\title{
Cuando el cuerpo ocupa la letra o la polifonía de voces. Sobre Los invasores de Egon Wolf en escena
}

\section{Alai Garcia Diniz}

Universidade Estadual do Oeste do Paraná (UNIOESTE) /Fundadora del “Núcleo de Estudos de Literatura, Oralidade e Outras Linguagens" (NELOOL/UFSC)

agadin@gmail.com

\author{
Beatrice Távora \\ Universidade Federal de Santa Catarina (UFSC) \\ tavorabeatrice@gmail.com
}

\section{José Ricardo Goulart}

Universidade do Estado de Santa Catarina (UDESC)

8ocoisas@gmail.com

\author{
Luiz Gustavo Bieberbach Engroff \\ Universidade do Extremo Sul Catarinense (UNESC)-Universidade Federal de Santa Catarina \\ (UFSC) \\ gus.biber@gmail.com
}

Fecha de recepción: 09/06/2018. Fecha de aceptación: 19/09/2018.

\section{Resumen}

Desde la lectura a la aplicación pedagógica, este artículo plantea el cruce de distintas perspectivas de un proceso de enseñanza de literatura hispánica en lengua extranjera, concebida como una experiencia interdisciplinar en dialogo con las artes escénicas. Con el uso de la metodología de enfoque por tareas, el objetivo final era testar la conexión de un curso de tercer grado (Letras/Español) con la recepción en otro nivel de enseñanza: la educación básica. El ensayo analiza el proceso con los no-actores, la performance y los informes estudiantiles de la performance de un texto dramático chileno - Los invasores, de Egon Wolff, a estudiantes de lengua española del nivel medio en una escuela pública de la ciudad de Florianópolis (Brasil), a partir de las voces de los actores involucrados en ese estudio.

Palabras clave

Los invasores Wolf

Enseñanza de literatura Teatro

Interdisciplinariedad 


\section{When the Body Occupies the Letter or the Polyphony of Voices: on Egon Wolff's Los invasores on stage}

\begin{abstract}
From reading to pedagogical application, this paper presents the intersection between different perspectives of a teaching process from a course in Foreign Language Literature (Spanish) designed by an interdisciplinary dialogue with experience in Performing Arts. Using a task-based methodology, the ultimate goal was to test the connection between the Undergraduate Literature Course (Literature/Spanish) and the Middle School. This paper analyzes the process, performance and presentation's report of a dramatic chilean text - Egon Wolff's Los invasores - to Spanish students in Florianópolis City (Brazil), from the voices and reflections of the different actors involved in this study.
\end{abstract}

\section{Introducción}

Las culturas pueden entenderse mejor unas con otras por medio de sus performances (Taylor, 2013:28). En base a esa reflexión y a algunas epistemologías de descolonización en la educación superior fue propuesto un encuentro activo entre los estudiantes, con el propósito de convertir parte de las clases de literatura hispánica en un proyecto interdisciplinario colectivo. La experiencia metodológica de un enfoque por tareas proponía la puesta en escena de una obra teatral que comprendería tres etapas con reglas específicas, conocidas por los estudiantes: el proceso (preparación - elección del texto y ensayo); la performance o espectáculo a los estudiantes de español de otro nivel de enseñanza y el informe final.

Puntos de partida fundamentales fueron, en primer lugar, recordar al grupo de estudiantes de lengua extranjera que uno de sus futuros roles sería lo de volverse docente de Lengua Española en la educación fundamental y, para eso, ofrecer la oportunidad de reflexionar sobre un proceso gradual de conocimiento personal y reconocimiento colectivo, con la apertura interdisciplinaria brindaba la posibilidad de aprender algo de las artes escénicas, no solo leyendo la dramaturgia hispánica, sino también incorporando la lengua en vivo con el juego de la performance que exige la mirada a los espectadores. Asimismo, creer que la performance de una obra teatral de otra cultura ofrece la oportunidad de aprendizaje intercultural, tanto para el grupo que representa la obra como para el público que la escucha; esto se logra por medio de la producción lingüística en la lengua meta con los giros y léxicos propios de aquel país de habla hispánica y que las reflexiones, paso a paso, obedecen a una experiencia distinta en la clase de literatura al tratar obras teatrales consideradas canónicas.

La metodología del enfoque por tareas que surge en los años 90 en el ámbito anglosajón consiste en proponer el uso de la lengua real como proceso de adquisición de competencias lingüísticas ${ }^{1}$. Ese método ampliado a la enseñanza de la literatura en enseñanza de lengua extranjera (ELE), al tiempo que implica aprendizaje incorporada de texto en la lengua meta, se aplica también a un propósito interdisciplinario de sobrepasar las fronteras disciplinarias entre las letras y las artes, atendiendo a una perspectiva intercultural. La duda sobre los límites impuestos por la epistemología basada en la razón moderna se aplica para establecer otros paradigmas que es la educación natural, como la indígena, que se ofrece por la corporalidad y como solidaridad comunitaria hacia la evaluación de un resultado de aprendizaje.

\section{Keywords}

Los invasores

Wolff

Literature Teaching

Theatre

Interdisciplinarity
1. Para conocer más sobre las bases de la metodología de enfoque por tareas, acceder a http://cvc.cervantes.es/aula/didactired/anteriores/ mayo_01/03052001.htm y http:// comprofes.es/content/el-enfoquepor-tareas-un-m\% $\mathrm{C}_{3} \%$ Agtodo-

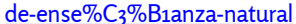


Al contemplar el ejercicio de la actuación como modo gradual de reconocimiento de un proceso colectivo e individual, se agregó a los dramas más conocidos de Lope de Vega, Tirso de Molina, Ramón María del Valle Inclán y Federico García Lorca, otros dramas menos conocidos por el hecho de que son latinoamericanos y llegan más difícilmente a Brasil. En ese curso se discutieron las obras Los invasores (1963) del chileno Egon Wolf y Los albañiles (1964) del mexicano Vicente Leñero.

En medio a los seminarios y discusiones del programa de Teatro Hispánico, se añadió un campo de experimentación con la colaboración de dos directores de teatro, egresos del curso de Artes Escénicas de la Universidad Federal de Santa Catarina (UFSC), un proceso de puesta en escena bajo el enfoque por tareas aplicado al curso de literatura con el objetivo de la práctica de un idioma extranjero (el castellano). Por medio de un plano concreto de construcción espectacular que se armaría a lo largo del semestre, con vistas a una performance final, se tomarían dos segmentos distintos de la educación en lengua extranjera: los futuros docentes (estudiantes de Letras) y los estudiantes de lengua española de la enseñanza media.

Esa acción pedagógica externa a la Universidad puede enseñarnos, entre otros aspectos, la necesidad de la lectura activa de un drama para adecuarlos a las circunstancias concretas de una performance. Vale decir que la elección de la obra Los invasores, realizada por los estudiantes de Letras, salió de entre otras dos propuestas: Don Juan Tenorio (1844) de José Zorrilla, y La eternidad y el vampiro (2007) de Emilio Ballesteros. Sin intervención de la docente, los criterios de decisión de los estudiantes tuvieron en cuenta al público joven que serían los receptores de la secundaria y que, de algún modo, expresaría el deseo de establecer un puente de comunicación con un imaginario más cercano a la realidad latinoamericana.

Cabe también observar que el propósito de proponer una experiencia concreta de los estudiantes de Letras en clave interdisciplinaria y en diálogo con el área de las Artes Escénicas proviene del hecho de tomar los archivos, previamente definidos como canon literario, como parte sospechosa, y proponer la acción colectiva de reorganizar dramatúrgicamente el texto escrito y, al pasarlo al cuerpo, crear la conciencia de la acción como parte de una vivencia en comunidad.

Al inventarse para sí la conducta de considerar el concepto de buen vivir, según lo propone Enrique Dussel (2012): El buen vivir, que recupera la noción quechua de proponer una ética distinta a la modernidad con su razón eurocéntrica. Sintéticamente, Dussel explica que sustantivar un verbo (vivir) y adjetivarlo (buen), de hecho, no se refiere a una buena vida, sino a "un modo de vivir en acción y para lograr el bienestar colectivo" ${ }^{2}$.

Por lo tanto, proponer que, además de evaluar a cada estudiante según su desarrollo subjetivo, parte de las clases proponían una metodología del enfoque por tareas, con el propósito de observar también qué acciones individuales aportaría el estudiante a un colectivo (el grupo de teatro). Este conjunto necesitaría de todos para convertir las tareas en acciones concretas sobre el texto, vestuario, sonido y demás aspectos de un espectáculo teatral ${ }^{3}$ para llegar a un final común: la performance a los estudiantes de la secundaria.

Con base en la acción y creación de repertorios, la búsqueda por otras racionalidades sería el fondo epistemológico y pedagógico como invitación a pensarse la educación superior en una universidad ubicada en el hemisferio sur. Es decir, leer un archivo (mapa, texto literario, restos arqueológicos, huesos) no como monumento $\mathrm{u}$ obra inmutable y duradera, sino como, sugiere Diana Taylor, desde la etimología de archivo que viene del griego y que, además de acoger el sentido de "lugar donde se

2. Extracto de la charla "El buen vivir”, de Enrique Dussel, en el Primer Encuentro del Buen Vivir (2012).

3. Entre los objetivos, no constaba la producción de escenario, vestuario u otro elemento visual. Se proponía un teatro despojado. No hubo ninguna clase de financiación, ni tiempo para buscarlo. 
guardan registros", arkhé significa también un comienzo, un primer lugar que sostiene el poder (2013:49). Es decir que, para Diana Taylor, hay que romper con el mito de que el archivo resiste al cambio. En el caso de los textos literarios canónicos (u obras teatrales), esto dependería de cómo se da la mediación de sus lectores. Se rompe con la sacralización de un texto canónico inmutable y se crea un repertorio. Si el archivo es un objeto, un documento, un texto; el repertorio no se confunde con su registro. Como performance o comportamiento ritualizado es efímero. El registro en video no se confunde con el momento de interacción entre los actores y los espectadores.

Desde la música hasta la formulación de Michael Bakhtin, la concepción de polifonía observa las múltiples conciencias que surgen en la novela de Dostoiewski que se mantienen en absoluta igualdad, sin someterse a una sola reflexión del narrador o autor. Ese trabajo detiene distintas voces que se combinan tan solo sobre la unidad de la experiencia, pero mantienen su perspectiva sobre el trabajo hecho ahora en cuatro miradas: la de la docente que propuso tal idea para un curso; la de dos egresados de las Artes Escénicas que dirigieron el proceso hasta la performance, la de una voz estudiantil que participó activamente de todas las etapas con la vivencia suya y la de algunas compañeras que autorizaron el uso de su material. Por lo tanto, a partir de esa introducción se ofrecen ahora las demás voces que configuran la experiencia, sea para operar otros temas o para proponer otros dispositivos, ya que una sola mirada no podría nunca dar cuenta de lo abarcado.

\section{La mirada desde las Artes Escénicas}

La posibilidad de trabajar en la dirección de un montaje teatral con no actores ${ }^{4}$, como auxilio del contenido práctico de la asignatura "Literatura Hispánica II", se presentó como un desafío para los directores, como también para los estudiantes. Nunca antes habíamos trabajado con estudiantes de otras áreas en un proceso creativo rumbo a un montaje final y ellos, a excepción de una de las alumnas, conocían el hacer teatral únicamente desde la perspectiva del espectador. Aceptamos el reto por creer en la interdisciplinariedad como base para nuestros trabajos y como fuerza impulsora de la creación artística, que enriquece experiencias mediante la adición de los aspectos de las distintas áreas del conocimiento. Los alumnos, en su gran mayoría, también lo aceptaron.

Los encuentros dirigidos a la práctica ocurrían inicialmente a cada quince días con duración aproximada de dos horas. En estos primeros momentos, nuestra intención era familiarizar los alumnos con el lenguaje teatral tras juegos y ejercicios basados en referencias como Viola Spolin, Augusto Boal y John Mowat ${ }^{5}$. En los primeros encuentros preferimos trabajar con juegos de improvisación oral, sin la utilización de expresión corporal, pues entendíamos que de esta manera podríamos establecer poco a poco una relación de confianza con los alumnos hasta que todos estuvieran cómodos lo suficiente para exponerse ante nosotros y sus compañeros. Además de esto, procurábamos participar de todas las dinámicas propuestas junto con los alumnos, pues, según Viola Spolin (2006:6), crear un ambiente de convivio basado en la igualdad entre todos, permite lograr la libertad y la expresión personal de los estudiantes. Esta estrategia resultó beneficiosa cuando percibimos que la timidez para quitarse las zapatillas, para hacer los estiramientos y las muecas durante el calentamiento vocal fue disminuyendo a medida que los encuentros se sucedían.

En algunos ejercicios que tenían la intención de explorar la creatividad, nos dimos cuenta de que había algunas dificultades para liberarse de lo real o crear cosas fantasiosas, pero, poco a poco, los participantes lograron relajarse y hacer el juego. En esta época, como posible sugerencia para la escenificación, los estudiantes leían una
4. Término acuñado por el director y dramaturgo Augusto Boal en los libros 200 Exercícios e Jogos para o Ator e o Não Ator com Vontade de Dizer Algo Através do Teatro y Jogos para Atores e Não Atores. Boal creía que el teatro es una actividad intrínseca del ser humano, que puede ser practicada por actores profesionales o no, en cualquier sitio.

5. Director inglés que dio talleres de Juegos Teatrales e Improvisaciones en Brasil el septiembre de 2012 y 2013. Una vez que participamos de los talleres en las dos ocasiones, utilizamos con los alumnos de Letras una parte de los conocimientos y algunos de los ejercicios realizados en el curso de estas vivencias. 
versión con recortes del texto Don Juan Tenorio (1844) de José Zorilla. Por eso, basados en esta versión, desarrollamos las dinámicas de uno de los encuentros. Dividimos la clase en grupos de tres o cuatro componentes (que crecía con la llegada de los demás estudiantes). Solicitamos que hablasen acerca de sus lecturas con el fin de recuperar la historia, mientras los demás grupos expresaban su acuerdo o su desacuerdo con lo contado. Todos deberían participar, aunque no hubieran leído (en esta situación deberían hacer una improvisación acerca de lo hablado por el estudiante anterior) y contar el mínimo de la obra.

Cuando iniciamos el segundo momento de la dinámica, en que los estudiantes deberían hablar acerca de las consideraciones hechas por los otros grupos, percibimos una divergencia entre todos acerca de la culpabilidad o inocencia de los personajes, lo que constituyó la mecha para provocar un debate. La conversación se transformó en discusión y tuvimos la idea de proponer un juicio. Divididos en dos grupos, uno haría la defensa y otro la acusación del personaje Isabela. Aclaramos que deberían hacer la defensa o la acusación inclusive en contra de sus posiciones personales. Los alumnos participaron del juego y defendieron el punto de vista del grupo, desarrollando argumentaciones para justificar su posición (defensa/acusación).

En este encuentro, la propuesta era un trabajo de reflexión y comprensión del texto. Cuando empezaron los debates, nos dimos cuenta de que estábamos ante un grupo de personalidades diferentes con algunas ideas en común. Tomamos este hallazgo para proponer el juego del juicio, en el cual los alumnos deberían expresarse con el uso de improvisaciones, pues, según los estudios de Jean Pierre Ryngaert:

La experiencia en la que la improvisación suele basarse no se limita a la afirmación del yo o de la afectividad del jugador. Ella también se extiende a una suma de experiencias del mundo de las que el sujeto es depositario y de las que se encuentran rastros en los guiones" (2009:93). ${ }^{6}$

La idea era fomentar improvisaciones a partir de distintos puntos de vista para evitar que posibles afectaciones (relacionadas con los personajes, los textos, los temas) pudieran interferir en las futuras decisiones durante el proceso.

La semana siguiente comenzamos las prácticas direccionadas al texto Los Invasores ${ }^{7}$. Siempre iniciábamos los encuentros con actividades de estiramiento y calentamiento. Para desarrollar el foco y la atención después del calentamiento, hacíamos un ejercicio en el cual, en círculo, uno de los alumnos decía su nombre y miraba hacia otro haciendo la transferencia del foco. Este estudiante, a su vez, debería hacer lo mismo en relación a otro compañero y, de esta manera, todos en su momento. En el universo de la obra, uno de los primeros ejercicios dividió la clase en dos grupos. Basados en el tema central e inspirados en los personajes, mitad de los alumnos debería asumir la posición de invasores y otra mitad de burgueses. Los invasores se quedaban en el centro de la clase, mientras los burgueses deberían cruzar la clase sin que los otros pudiesen alcanzarlos. Los que eran capturados se convertían en invasores. Mientras hacían este ejercicio, solicitamos que los alumnos enseñasen, además de su forma de caminar y correr, los gestos característicos de los dos grupos.

Al final del encuentro, la clase fue dividida en cuatro grupos que, a partir del texto, improvisaron una escena, de acuerdo con su predilección. Por casualidad, los grupos utilizaron trechos del primer acto en el cual ocurre el primer embate del personaje rico con el invasor. La razón para que esto ocurriera puede tener origen en distintos factores, pero podemos apuntar dos: la mayoría no había logrado leer el texto completo o bien el inicio de la obra era un momento adecuado para la presentación de algunos de los protagonistas.
6. [“A vivência na qual normalmente a improvisação se apoia não se reduz à afirmação do eu ou da afetividade do jogador. Ela se estende igualmente a uma soma de experiências do mundo das quais o sujeito é depositário e das quais se encontram vestígios nos roteiros."]

7. Los Invasores se estrenó en Chile en 1963, en el Instituto de Teatro de la Universidad de Chile, con dirección de Víctor Jara. Narra la historia de una familia burguesa que tiene su casa invadida por personas sin hogar, conocidos en el texto como "los del otro lado del río". Habla del tema de la desigualdad social en el país en aquella época, lo que es una realidad en la actualidad en el mundo. Causó reacciones distintas y polémicas. 
El desarrollo de las escenas, en su mayor parte, presentaba dificultades. Los estudiantes no enunciaban el texto de manera bien articulada, se alejaban hacía el fondo de la clase (quizás por timidez, como a ocultarse de los demás que los miraban) y no respondían a los estímulos originados de los otros. En estos primeros ejercicios referentes al trabajo textual, fue posible constatar que la dificultad del proceso se debía a que en los participantes no estaba claro el rigor exigido por el teatro; los actores no tenían conocimiento del texto que se proponían a escenificar, no trabajaban bien colectivamente y no atendían a los horarios predefinidos de los encuentros. No obstante, esta situación no era generalizada: algunos de los participantes se destacaban por el hecho de que mostraban más desinhibición, responsabilidad con la puntualidad y haber realizado una lectura más profunda de la obra. Sin embargo, cuando se trata de un trabajo colectivo, la ausencia, el retraso o la desatención de uno influye en el resultado colectivo.

Con el desarrollo del proceso, el perfil de cada alumno se fue evidenciando y, desde entonces, reconocemos en el grupo los cuatro elementos que dificultan el desarrollo del juego, descritos por Ryngaert (2009:45-52), en su libro Jogar, Representar: la inhibición relacionada con la timidez o con un bloqueo causado por la mirada del otro o su propia autocritica; la extroversión que comprende un exceso de manifestación del participante; la negación del juego que es la dificultad de liberarse de lo real y el savoirfaire limitad, manierismos que no permiten el descubrimiento del lenguaje teatral.

A partir de estas identificaciones, buscamos disminuir las dificultades de entendimiento y de dinámica individuales de cada uno de los jugadores, en una tentativa de mezclar el grupo en una unificación del lenguaje teatral intentando convencer de que, en el momento de la escena, era el grupo el que conseguiría hacerlo. El grupo estaba compuesto por todos los jugadores, cada cual con sus opiniones y deseos pero, en aquel momento, debería haber un punto común: la performance marcada desde el inicio del semestre.

En el transcurso de las reuniones, los participantes comenzaron a entender un poco más la dinámica teatral y la importancia de unidad del grupo para este tipo de trabajo $y$, entonces, en realidad, empezaron a entrar en el juego: formaban grupos de estudios para memorizar y hablar el texto e, inclusive, ayudar en la construcción estética de la escena. Cada uno tomaba su espacio dentro de una construcción colectiva, cuando estimulaba a los compañeros alejados de las necesidades del grupo y cuando tomaba la actitud de realizar ensayos fuera de los horarios de los encuentros semanales. Podemos ilustrar con la idea que surgió de una de las participantes: en uno de los grandes momentos de la escenificación en el cual los actores, mezclados con el público, se levantaban de diferentes partes de la audiencia a cantar juntos la canción $A$ Desalambrar, del cantante chileno Víctor Jara.

La aproximación de la fecha de estreno hizo que todos estuvieran aún más motivados, ya que ninguno de ellos quería mostrarse frágil en escena delante de la audiencia. A pesar de la deserción repentina de algunos colegas unos pocos días antes del estreno, los que permanecieron en el grupo se volvieron más unidos y seguros. Preocupados con la presentación, las ideas comenzaron a surgir, quizás un poco tarde, pues ya no habría tiempo para nuevos experimentos. Teníamos que apostar en el material conquistado durante el semestre y este material representó la construcción conjunta de los estudiantes, de no actores que se retrasaban, que se ausentaban, que abandonaban. Ello fue posible porque los demás resistieron, superaron sus miedos, ansiedades y limitaciones. Resistieron y lograron llegar a la meta final. Tal vez para muchos de ellos haya sido una experiencia única, pero a sabiendas, podemos definirlo como transformadora. Al menos para los que se subieron al escenario el 27 de noviembre de 2013. 
Para nosotros, los directores, este proceso creativo ha sido una constante búsqueda de diferentes maneras y medios para llevar al grupo a los personajes propuestos por el dramaturgo Egon Wolff. Al final de cada encuentro discutíamos lo que se presentaba y la unión del grupo, a pesar de que se consolidó más tarde, colaboró para que la obra fuera presentada en el día establecido.

Creemos que el trabajo realizado nos permitió hacer una revisión de la didáctica para el trabajo en grupo. Los procedimientos adoptados, basados en la bibliografía especificada para el hacerteatral no siempre son aplicables en su integralidad. Debemos estar abiertos a nuevas propuestas, ideas y estímulos procedentes del grupo para lograr un resultado adecuado. Tal vez este fue el mayor aprendizaje durante el proceso: una constante reflexión sobre cómo lograr extraer del grupo un mejor desarrollo, una vez que fórmulas y sistemas no siempre funcionan en la práctica como se describe en los libros.

\section{La voz de las Letras}

La asignatura de Literatura Hispánica II es requisito obligatorio del curso de Licenciatura en Letras Españolas, ofrecido por la UFSC con el objetivo de fomentar el estudio de los momentos significativos del teatro hispánico e hispano-americano y, además de las lecturas y análisis de obras representativas de este género, exige la aplicación de los conocimientos adquiridos en el montaje de una obra teatral en español. Así, a lo largo de todo el segundo semestre 2013, bajo la orientación de la profesora y en dos encuentros semanales de noventa minutos, los alumnos fueron estimulados a investigar y pensar acerca del teatro hispánico e hispano-americano con la perspectiva de la puesta en escena de una obra teatral.

El grupo, compuesto por estudiantes con edades entre 20 y 50 años, oriundos de distintos países y con un dominio heterogéneo del idioma, tras una toma de decisión conjunta con la profesora, acordó en planificar el proyecto de escenificación que quedó dividido en tres etapas distintas. En la primera etapa, denominada proceso, fueron agrupadas y observadas la frecuencia y puntualidad de los participantes, la iniciativa y memorización del texto, la participación activa en el escenario, el auxilio en distintas tareas y resolución de conflictos y la elaboración de un diario de campo para el informe de la etapa final. La segunda etapa, denominada performance estaba relacionada con el desempeño en la puesta en escena y el informe compuso la tercera etapa y comprendió el relato personal de cada uno de los participantes en todo el proceso.

Como forma de corroborar la importancia de la experiencia y el proceso de construcción de sentido para los participantes, serán presentados algunos de los testimonios más significativos de los informes que serán identificados con el uso de letras (A hasta E), de manera a preservar identidades.

Lo primero entonces que se ha de aclarar es el abandono de la idea caricaturesca y sesgada con que se habla de lo comunicativo, como apunta Llobera, que a menudo se presenta como una aproximación a la enseñanza de lengua que se opone a la gramática y a la literatura para afirmar, con este autor, que "la literatura es una de las formas más exigentes de comunicación escrita y por tanto ineludible en una aproximación comunicativa desarrollada de la enseñanza de lenguas" (1995:5).

De esa manera, la elección de un texto literario del género teatral fue una estrategia que hizo posible la reflexión acerca de la teoría y de la práctica, a fin de fomentar en los participantes destrezas comunicativas que contribuyeron a su propio desarrollo 
mediante la adquisición de conocimientos y cultura en general. Para eso, luego en las primeras clases, la profesora estimuló a todos para que buscasen algunos textos para la performance, lo que provocó una gran preocupación, pues era necesaria la elección de un escrito, cuyo tema y lenguaje despertase el interés de los espectadores, estudiantes de secundaria con edad entre quince y diecisiete años. En los relatos de los participantes/actores es posible identificar esta preocupación cuando se lee:

Tras la conducción de la profesora tuvimos contacto con varios textos y algunos videos de escenificaciones hechas por grupos distintos alrededor del mundo. Algunas semanas se pasaron hasta que el grupo tomó la decisión de trabajar el texto Los Invasores porque el tema parecía más apropiado para el público de estudiantes de secundaria y también porque eran muchos los personajes y así habría oportunidad para la participación de todos (alumna E).

(...) había como posibilidad de montaje otras dos piezas de teatro - una de ellas era La eternidad y el vampiro, de Emilio Ballesteros. El proceso de elección del texto duró poco más de un mes y por fin la mayoría manifestó el interés por Los invasores (alumna A).

La lectura del texto Los Invasores y del contexto en que fue escrito permitió la ampliación del léxico, la observación de la sintaxis y de la morfología del español, así como una aproximación a una realidad histórica y a una cultura distinta. Al mismo tiempo, posibilitó el acercamiento a las características del género, cuya lectura exige operaciones mentales complejas, ausentes en otros géneros, que contribuyó en gran manera para la construcción de los personajes tras la percepción de sus rasgos fundamentales, sean físicos o psicológicos.

Juntamente con la elección del texto, otro desafío que se impuso desde el principio fue la superación de los límites individuales de los alumnos, dado que la mayoría nunca había participado de este tipo de propuesta y muchos no se creían capaces de llevar a cabo esta nueva experiencia. Todos estaban temerosos y manifestaran este sentimiento en los informes:

(...) Cuando la profesora nos contó su propuesta de hacer teatro, por poco no desistí. Le dije que no lograba memorizar el texto (alumna B).

(...) nadie creía que pudiera hacer una presentación para una escuela (...), sufrimos muchísimo, quedamos días sin dormir, sin almorzar para poder entrenar un poco más. Pero, nada iba bien, algunos alumnos renunciaron la presentación, pues ciertamente no creían que éramos capaces de desarrollar la obra (Sic) (alumna C).

Para auxiliar en este desafío, fue decidido que participarían dos directores, egresados del curso de teatro de la UFSC, y, cuando de hecho empezaron las actividades prácticas propuestas por ellos, el grupo fue desarrollando la consciencia de colectividad a partir de la individualidad o sea, percibiéndose individualmente, cada uno de los participantes se dio cuenta de que ocupaba un determinado espacio, de que poseía determinadas características que lo hacían único y que podrían ser utilizadas en la construcción del todo.

Cuando la primera oportunidad de reparto de los roles, la lectura del texto y la consciencia de las características individuales proporcionó a cada uno de los alumnos un contacto con los personajes que se fue profundizando con el paso del tiempo y ayudando en la superación de la etapa inicial en que todos estaban temerosos. Además de esto, el contacto con el personaje, la interiorización de sus características, intensificó la posibilidad de construcción de un discurso consistente desde el punto de vista de la 
lógica, estimulando los mecanismos de competencia discursiva dentro del proceso de aprendizaje del idioma cuando cada uno, en el momento apropiado, debería tratar de hacer llegar al grupo, con cohesión y coherencia, los aportes capaces de fortalecer los rasgos peculiares de cada personaje, tras la capacidad de argumentación. Los relatos son significativos en cuanto a este punto:

Los ejercicios propuestos eran, entonces, direccionados a la construcción de los personajes: ¿quién eran ellos?; ¿cómo se comportaban?; ¿cuáles sus conflictos?; ¿de qué manera las distintas personalidades impactaban, corporalmente, en cada uno? A partir de tales cuestiones, intenté "dar vida", por medio del lenguaje corporal, a Toletole (...). Para mí, la chica era una metáfora lírica que personificaba la propia injusticia (alumna A).

Entre las actividades de calentamiento (warm up) había una en la que caminábamos por el aula imaginando nuestro personaje. Después, elegimos una frase de nuestro personaje para decirla en voz alta. Todos al mismo tiempo y muchas veces. Cada uno entonces iba al centro y decía su frase de tres maneras distintas (...). Nos pusimos en grupos para ensayar la obra y elegí el niño (que después transformé en niña) que no habla mucho (alumna B).

A esta altura surgió el problema de la extensión del texto y de los parlamentos de los personajes que se resultaban demasiado largos para estudiantes principiantes y para el tiempo que había para estudiarlos. La decisión de los directores entonces se orientó en el sentido de hacer cortes para disminuir los turnos de habla y las intervenciones de cada participante, aunque siempre respetando el texto original. También, otra decisión importante, fue la escenificación por bloques de manera en que el mismo personaje fuera representado por estudiantes/actores distintos, caracterizados por un elemento común. Así, por ejemplo, para el personaje denominado Pietá fueron designadas tres alumnas diferentes que participaron en momentos secuenciales identificadas por un collar de fácil remoción. Para cada personaje fue elegida una vestimenta distinta con la función de permitir que el público hiciera la identificación a lo largo de toda la performance.

Otros dos recursos quedaron incorporados: la narración, tras la presencia de una alumna que presentaría el texto e introduciría los actos; y la canción que, en la apertura, integraría el público y los actores a la vez que estos iniciarían la performance sentados entre la audiencia, ingresando lentamente al por dos entradas laterales mientras la melodía sonaba. La canción $A$ Desalambrar, del cantante chileno Víctor Jara trajo dinamismo en la introducción del tema central por ser representativa del mismo periodo histórico del texto de Wolff.

Con el paso del tiempo y la disciplina del grupo, el trabajo asumió una nueva dinámica que fortaleció la interiorización de los personajes y el trabajo individual de memorización de parlamentos. En este punto, la presencia de la profesora fue fundamental como elemento motivador, porque era ella quien miraba el trabajo ya hecho desde afuera y contribuía destacando positivamente lo que iba bien y cuestionando el grupo acerca de lo que podría ser mejorado.

La intensificación de los ensayos en ese momento exigió un esfuerzo mayor, pues las actividades iniciaban con los ejercicios de preparación y culminaban con la escenificación haciendo que, además de la exigencia de expresión oral, se agregaran otros elementos pensados para la performance. La competencia estratégica fue intensamente trabajada en las improvisaciones, ya que eran necesarias interacciones comunicativas significativas y realistas para que la dinámica de la obra funcionase. En muchos momentos, los alumnos tomaban el texto original como base de la escenificación 
pero, como la memorización no funcionaba muy bien, buscaban otros recursos en el idioma para expresar la misma idea, activando conocimientos previos interiorizados, buscando recursos interdisciplinares y habilidades sedimentadas para solucionar cuestiones no previstas.

La presencia constante y el compromiso del grupo son aspectos que merecen ser destacados en este punto, pues todo el trabajo solo caminaba bien si todos estaban en la misma sintonía. La actuación de uno dependía de la actuación del otro. El conjunto era lo que importaba considerando que la participación de cada personaje estaba interrelacionada con otro, en un juego escénico donde el resultado dependía de la integración de todos. El relato de los alumnos apunta para este hecho. Veamos:

A esta altura del proceso, las personas estaban todas (o casi todas) comprometidas con la importancia de hacer una buena puesta en escena. Y era visible el desarrollo de algunos - así como el hecho de que el texto aún no estaba clavado en otros. Esto, claro, comprometió un poco el resultado final, porque no hay como hacer teatro sin el envolvimiento total (conocimiento del texto y de todos los personajes, memorización del habla de su personaje, preocupación con la marcación del espacio teatral) del conjunto de personas (alumna A).

El estudio no era suficiente porque mi habla dependía del habla de mi compañero de escena. Era eso lo que yo tardé en percibir porque de acuerdo con lo que él hablaba mi texto sonaba distinto. Y así era para todos, estábamos todos en un entramado, unos colgados a los otros y cuando todos estaban conscientes de esto sentí una mejora, un poco más de seguridad. Era necesario entonces que estuviéramos juntos, muchas horas durante muchos días para que cada uno estuviera seguro lo suficiente (alumna E).

A medida en que se aproximaba la fecha de presentación se realizó un ensayo general en el teatro, lo que posibilitó la percepción del espacio escénico y de todo lo que significaba ocupar este espacio: las marcaciones de los movimientos, los elementos que integraban la escena, los sitios que marcaban la entrada y salida de los personajes, los efectos sonoros e inúmeros otros elementos que aportarían seguridad para los participantes/actores a la hora de la performance. Estar en este espacio y actuar hizo con que el proyecto se concretara, aunque algunos problemas insistían en aparecer.

Por decisión de los directores y de la profesora, se planteaba en algunos momentos la interacción con el público, lo que surgió como solución apropiada a la necesidad en el palco de una ventana por la cual los personajes miraban hacia el exterior de la casa donde se suponía que sucedían los acontecimientos. Algunos carteles fueron el recurso para que el público se manifestara en los momentos apropiados.

Finalmente, el día del estreno trajo las aprensiones normales en todos los participantes pero, todo se llevó adelante de manera feliz. Las escenas que fueron mejor presentadas fueron realizadas por los alumnos más comprometidos durante todo el proceso y la experiencia dejó huellas en todos, tal como quedó registrado en los informes:

Para mí, la experiencia fue fantástica. No sólo porque me gusta mucho el teatro, sino sobre todo porque pude, después de tantos años, experimentarlo como vivencia (alumna $\mathrm{A}$ ).

Me gustó el resultado. Me sorprendí con la actuación de algunos de los colegas (Sic) (alumna B). 
A mí me gustó mucho la interacción de los estudiantes durante la puesta en escena y, al final de la presentación, como si fuera el teatro de Augusto Boal con la interacción entre los personajes y los estudiantes caminando por medio de ellos junto a las sillas, hablando, sacando fotos; todo eso es muy importante para valorar el trabajo de los actores, los personajes, la presencia del pueblo, la pieza, la obra y el autor (Sic) (alumna C).

\section{Consideraciones finales}

Así, tras este breve recorrido en el cual fueron presentadas algunas peculiaridades del teatro como práctica didáctica en el proceso de enseñanza/aprendizaje de lengua extranjera, es posible afirmar, como consideraciones finales, que en el curso de licenciatura uno debe tratar de elaborar recursos creativos para colaborar con la diseminación del conocimiento. Trabajos como este pueden demostrar a los principiantes las inmensas posibilidades de incentivo a la apropiación significativa del conocimiento.

Al incorporar un archivo, el estudiante amplia los horizontes de su lectura por entenderse como mediador. Cuando el cuerpo ocupa el espacio de las letras, se escuchan voces en vivo y eso es lo hic et nunc que no puede volver a repetirse. Con el proceso de escenificación de un texto en que el cuerpo (colectivo) invade el espacio de las lecturas individuales, el estudiante aprehende la posibilidad de releer una obra con una propuesta práctica de sociabilidad, invención, solidaridad, convivencia entre sus pares para llegar a la performance. 


\section{Q Bibliografía}

» Bakhtin, M. (2008). Problemas da poética de Dostoiévski. Rio de Janeiro: Forense Universitária.

» Banciotti, M. C. y Ortecho, M. (2013). "La noción de performance y su potencialidad epistemológica en el hacer científico social contemporáneo". Tabula rasa, 2013 (19), 119-137.

» Boal, A. (1997). 200 Exercícios e jogos para o ator e o não ator com vontade de dizer algo através do teatro. Rio de Janeiro: Civilização Brasileira.

»Boal, A. (1998). Jogos para atores e não atores. Rio de Janeiro: Civilização Brasileira.

»Dussel, E. (2012). Enrique Dussel - Primer Encuentro del Buen Vivir - El estado como campo de lucha. [archivo de video] Consultado el 10 de febrero de 2014 en $<$ http://www.youtube.com/watch?v=ieRwulurppo $>$.

»Llobera, M. (1995). Competencia Comunicativa: documentos básicos en la enseñanza de lenguas extranjeras. Madrid: Edelsa Grupo Didascalia.

» Ryngaert, J. P. (2009). Jogar, representar. São Paulo: Cosac Naify.

»Spolin, V. (2006). Improvisação para o teatro. São Paulo: Perspectiva.

» Taylor, D (2013). O arquivo e o repertorio - performance e memoria cultural nas Américas. Belo Horizonte: Editora UFMG.

»Wolf, E. (1963). Los invasores. En: Biblioteca Digital Terra Nova. [en línea]. Consultado el 29 de enero de 2014 en http://bibliotecadigitalterranova.wikispaces. com/file/detail/Los+invasores+(Egon+Wolff).pdf 